\title{
Fotoperíodo sobre parâmetros fisiológicos relacionados ao estresse em alevinos de tilápia-do-nilo
}

\author{
[Photoperiod on physiological parameters related to stress in Nile tilapia fingerlings] \\ G.C. Veras $^{1}$, L.D.S. Murgas ${ }^{2}$, M.G. Zangeronimo ${ }^{2}$, P.V. Rosa ${ }^{2}$, \\ J.A.S. Leon $^{2}$, A.L. Salaro ${ }^{3}$ \\ ${ }^{1}$ Universidade Federal do Pará -UFPA - Bragança, PA \\ ${ }^{2}$ Universidade Federal de Lavras -UFLA - Lavras, MG \\ ${ }^{3}$ Universidade Federal de Viçosa -UFV - Viçosa, MG
}

\begin{abstract}
RESUMO
Com este trabalho objetivou-se avaliar a influência de diferentes fotoperíodos sobre parâmetros fisiológicos relacionados ao estresse em alevinos de tilápia-do-nilo (Oreochromis niloticus). Foi utilizado um delineamento inteiramente casualizado, com cinco tratamentos $(0 \mathrm{~L} ; 6 \mathrm{~L} ; 12 \mathrm{~L} ; 18 \mathrm{~L} ; 24 \mathrm{~L})$ e quatro repetições, sendo o aquário a unidade experimental. Cento e sessenta alevinos de tilápia, $3,21 \pm 0,05 \mathrm{~g}$ e $4,35 \pm 0,07 \mathrm{~cm}$, foram distribuídos em 20 aquários de 20 litros, em sistema de recirculação com temperatura controlada em $27^{\circ} \mathrm{C}$ e densidade de oito peixes por aquário. Por um período de 75 dias, a alimentação foi realizada duas vezes ao dia com ração extrusada contendo $40 \%$ de proteína bruta. Ao final do período experimental, os dados de crescimento, sobrevivência, cortisol, glicose e hematócrito foram submetidos à análise de variância e, posteriormente, em caso de significância $(\mathrm{P}<0,05)$, foi realizado um teste Tukey a 5\% para comparação das médias. A manipulação do fotoperíodo demonstra não alterar a homeostase dos alevinos, uma vez que não há diferença sobre a sobrevivência, glicose sanguínea, hematócrito e cortisol plasmático $(\mathrm{P}>0,05)$.
\end{abstract}

Palavras-chave: cortisol, glicose, homeostase, Oreochromis niloticus

\begin{abstract}
The objective of this study was to evaluate the influence of different photoperiods on physiological parameters related to stress in Nile tilapia fingerlings (Oreochromis niloticus). A completely randomized design with five treatments $(0 \mathrm{~L}, 6 \mathrm{~L}, 12 \mathrm{~L}, 18 \mathrm{~L}, 24 \mathrm{~L})$ and four replications was used, and the aquarium as the experimental unit. One hundred and sixty tilapia fingerlings, $3.21 \pm 0.05 \mathrm{~g}$ and $4.35 \pm 0.07 \mathrm{~cm}$ were distributed in 20 aquariums of $20 \mathrm{~L}$ in a recirculation system with temperature controlled at $27^{\circ} \mathrm{C}$ and density of eight fish per aquarium. Feeding was performed twice daily with extruded feed containing $40 \%$ crude protein, for a period of 75 days. At the end of experimental period, the data of survival, cortisol, glucose and hematocrit were subjected to analysis of variance and, subsequently, in case of significance $(P<0.05)$ a Tukey test 5\% was performed to compare means. Manipulation of photoperiod shows no alteration in the homeostasis of juveniles, since there is no difference in survival, blood glucose, hematocrit and plasma cortisol $(P>0.05)$.
\end{abstract}

Keywords: cortisol, glucose, homeostasis, Oreochromis niloticus

\section{INTRODUÇÃO}

Grande parte das alterações ambientais pode agir como uma potencial fonte de estresse nos peixes, o que pode ser visualizado nas alterações hormonais, concentrações de determinados

Recebido em 12 de junho de 2012

Aceito em 25 de junho de 2013

E-mail: galileu@ufpa.br substratos no plasma e/ou por alterações no parâmetro de eritrócito (Barton, 1997; Biswas et al., 2004, 2006b). A exposição aos agentes estressores pode ser classificada como aguda ou crônica. O estresse agudo ocorre geralmente durante o manejo, como em situação de captura, biometria e transporte. O estresse crônico é 
ocasionado devido à exposição do animal a longos períodos em situações estressantes, o que pode levar a baixo crescimento, baixa reprodução e deficiência do sistema imunológico (Wendelar Bonga, 1997). Sendo assim, quando os mecanismos de respostas são frequentemente exigidos, as respostas do organismo podem prejudicar a saúde do animal (Barton e Iwama, 1991).

Por um longo período, têm-se estudado a influência dos fatores ambientais sobre os peixes (Boeuf e Bail, 1999). Dentre esses fatores, o período de luz, ou seja, o fotoperíodo, atua como um sincronizador do ritmo endógeno, influenciando sobre a atividade locomotora, o crescimento, as taxas metabólicas, a pigmentação corpórea, a maturação sexual e a reprodução de peixes teleósteos (Biswas et al., 2002; Biswas e Takeuchi, 2002; Boeuf e Bail, 1999; El-Sayed e Kawanna, 2004; Trippel e Neil, 2003).

Apesar de a manipulação do fotoperíodo alterar níveis de determinados hormônios em peixes, o estresse pouco vem sendo demonstrado como consequência aparente desse fator (Pickering e Pottinger, 1983; Audet et al., 1986; Biswas et al., 2004). No entanto, durante a larvicultura de algumas espécies, regimes longos ou constantes de luz têm demonstrado efeitos negativos em seu desenvolvimento, como nos processos de inflação da bexiga natatória e deformações ósseas (Villamizar et al., 2011). Sendo assim, generalizações quanto ao período de luz devem ser evitadas, procurando respeitar a preferência do fotoperíodo para cada espécie e sua fase de desenvolvimento.

A tilápia-do-nilo (Oreochromis niloticus) destaca-se pelo rápido crescimento em cultivo intensivo, por ser uma espécie rústica, além de ser uma das espécies mais cultivadas no mundo. Diversas pesquisas buscam continuamente novas tecnologias com o intuito de melhorar o sistema de produção dessa espécie. Estudos anteriores demonstraram que diferentes regimes de fotoperíodo podem ser utilizados para estimular o desempenho do crescimento em tilápia-do-nilo (Biswas et al., 2002; Biswas e Takeuchi, 2002; Rad et al., 2006; El-Sayed e Kawanna, 2004; Bezerra et al., 2008; Cruz e Brown, 2009). No entanto, seria precipitado propor esses fotoperíodos como ideais para a criação dessa espécie sem uma análise mais criteriosa de como o fotoperíodo poderia afetar os parâmetros relacionados ao estresse.

Sendo assim, com este trabalho objetivou-se avaliar a influência de diferentes fotoperíodos sobre os parâmetros relacionados ao estresse em alevinos de tilápia-do-nilo.

\section{MATERIAL E MÉTODOS}

O experimento foi realizado no Laboratório de Fotoperíodo de Peixes do Setor de Fisiologia e Farmacologia do Departamento de Medicina Veterinária da Universidade Federal de Lavras, MG, por um período de 75 dias.

Utilizou-se um delineamento inteiramente ao acaso, com cinco tratamentos, ou seja, diferentes fotoperíodos $(0 \mathrm{~L} ; 6 \mathrm{~L} ; 12 \mathrm{~L} ; 18 \mathrm{~L} ; 24 \mathrm{~L})$, e quatro repetições, sendo o aquário a unidade experimental.

Foram utilizados 160 alevinos de tilápia-do-nilo, com peso e comprimento iniciais de $3,21 \pm 0,05 \mathrm{~g}$ e $4,35 \pm 0,07 \mathrm{~cm}$, respectivamente, que foram distribuídos aleatoriamente na densidade de estocagem de oito peixes por aquário.

O experimento foi conduzido em 20 aquários de 20 litros, em sistema de recirculação de água, com temperatura controlada por termostato. Os parâmetros de qualidade da água - temperatura $\left(27,2 \pm 0,99^{\circ} \mathrm{C}\right), \quad \mathrm{pH}(6,95 \pm 0,05)$ e oxigênio dissolvido $(7,35 \pm 0,41 \mathrm{mg} / \mathrm{L})$-foram monitorados diariamente, enquanto a concentração de amônia $(0,12 \mathrm{mg} / \mathrm{L})$ foi monitorada uma vez por semana.

Grupos de quatro aquários foram mantidos isoladamente sob sistema de iluminação controlado por temporizadores individuais e uma lâmpada fluorescente de $20 \mathrm{~W}$ com constante intensidade de 1200 lx sobre a superfície da água. Todas as lâmpadas, dos respectivos fotoperíodos, eram acesas às $7 \mathrm{~h}$, exceto os programas de luz em que os peixes foram mantidos em regime de 24 horas de luz e escuro, cujas lâmpadas permaneciam ligadas e desligadas, respectivamente, por todo período experimental.

De acordo com metodologia descrita por Larson et al. (2004), para iluminação do ambiente externo do laboratório, foi utilizada uma lâmpada de coloração vermelha de $60 \mathrm{~W}$ que era acesa 
durante a alimentação, biometria, limpeza dos aquários e coleta de sangue.

Os peixes foram alimentados duas vezes ao dia, nos horários de $9 \mathrm{~h}$ e de $17 \mathrm{~h}$, com ração comercial extrusada contendo $40 \%$ de proteína bruta e peletes de dois milímetros de diâmetro. A quantidade de ração fornecida foi $5 \%$ do peso vivo durante os primeiros 15 dias e, posteriormente, $3 \%$ do peso vivo até o final do experimento. As biometrias foram realizadas em intervalos de 15 dias para a correção do fornecimento de ração.

Ao final do experimento, os peixes foram mantidos em jejum por um período de 24 horas. Em seguida, os animais foram anestesiados em 2-fenóxietanol $(0,6 \mathrm{~mL} / \mathrm{L})$ e, por meio de seringa tipo insulina, retirou-se aproximadamente $1 \mathrm{~mL}$ de sangue por punção cardíaca para análise de glicose, cortisol e hematócrito.

A glicose sanguínea foi avaliada em cinco peixes de cada aquário $(\mathrm{n}=20)$, com uma pequena alíquota de sangue através de aparelho digital de glicose (kit para análise de glicose humana - Accu Chec Advantage II - Roche ${ }^{\circledR}$ ). Para realização do hematócrito, também foram utilizados cinco peixes por aquário $(n=20)$.
O sangue destes foi transferido para tubos microcapilares heparinizados que foram centrifugados por $5 \mathrm{~min}$ a $12000 \mathrm{rpm}$. O restante foi armazenado em microtubos tipo eppendorfs, centrifugados a $3000 \mathrm{rpm}$ por 15 minutos. Em seguida, retirou-se o plasma, que foi etiquetado e armazenado também em microtubos tipo eppendorfs a $-80^{\circ} \mathrm{C}$, para posteriores análises de cortisol através da técnica de imunoensaio enzimático direto (Kit ELISA para análise de cortisol humano - Diagnostics Biochem Canada Inc. $\left.{ }^{\circledR}\right)$. Para análise de cortisol, foram utilizadas amostras de plasma de quatro peixes por aquário para cada fotoperíodo $(\mathrm{n}=16)$.

Com o auxílio do programa para análise estatística Sisvar, as variáveis foram analisadas primeiramente por uma análise de variância, teste $\mathrm{F}$, e, quando verificadas diferenças significativas entre os tratamentos $(\mathrm{P}<0,05)$, as médias foram comparadas pelo teste Tukey a $5 \%$ de probabilidade.

\section{RESULTADOS E DISCUSSÃO}

Não houve efeito do fotoperíodo sobre a taxa de sobrevivência, glicose sanguínea, hematócrito e cortisol de alevinos de tilápia-do-nilo $(\mathrm{P}>0,05)$ (Tab. 1).

Tabela 1. Efeito do fotoperíodo sobre a sobrevivência, hematócrito, glicose e cortisol em alevinos de tilápia-do-nilo

\begin{tabular}{|c|c|c|c|c|c|c|}
\hline \multirow{2}{*}{ Variáveis } & \multicolumn{5}{|c|}{ Fotoperíodo (horas de luz) } & \multirow[b]{2}{*}{ CV (\%) } \\
\hline & 0 & 6 & 12 & 18 & 24 & \\
\hline $\mathrm{TS}^{(1)}$ & $87,5 \pm 10,21$ & $84,63 \pm 15,27$ & $93,75 \pm 7,22$ & 100,00 & $96,88 \pm 6,25$ & 10,00 \\
\hline $\mathrm{Hem}^{(2)}$ & $37,07 \pm 7,36$ & $35,54 \pm 5,32$ & $35,82 \pm 4,41$ & $35,88 \pm 6,04$ & $37,60 \pm 5,35$ & 13,95 \\
\hline $\mathrm{Gli}^{(3)}$ & $39,30 \pm 9,21$ & $36,04 \pm 5,14$ & $37,48 \pm 7,76$ & $35,30 \pm 8,21$ & $38,62 \pm 11,48$ & 16,35 \\
\hline $\operatorname{Cort}^{(4)}$ & $76,30 \pm 15,00$ & $85,00 \pm 4,00$ & $83,75 \pm 13,00$ & $73,80 \pm 10,00$ & $71,30 \pm 19,00$ & 17,12 \\
\hline
\end{tabular}

Assim como a presente pesquisa, outros estudos com tilápia (Biswas e Takeuchi, 2003; El-Sayed e Kawanna, 2004) e juvenis de tambaqui Colossoma Macropomum (Mendonça et al., 2009) também não demonstraram efeito do fotoperíodo sobre a taxa de sobrevivência desses animais. No entanto, Shan et al. (2008), em estudos com larvas de Miiuy croaker Miichthys miiuy, demonstraram uma pior taxa de sobrevivência no tratamento de $0 \mathrm{~L}$, havendo
$100 \%$ de mortalidade após o sétimo dia de vida das larvas. Segundo os autores, a alta mortalidade pode ser atribuída à baixa habilidade dessas larvas em encontrar o alimento em ambientes com ausência de luz. Além disso, segundo Holmes e Gibson (1986), para espécies classificadas como predadoras visuais, a exposição e o movimento da presa são estímulos fundamentais para detecção e reconhecimento do alimento. Assim, peixes que fazem uso da visão 
como recurso para capturar seu alimento poderiam ser prejudicados quando em ambientes onde o alimento se distribui em locais pouco iluminados, ou onde o período de luz durante o dia é curto (Dabrowski, 1975).

Por outro lado, Adewolu et al. (2008) demonstraram que alevinos de bagre africano Clarias gariepinus apresentaram melhor taxa de sobrevivência quando criados sob um fotoperíodo de 0L. Segundo os mesmos autores, a espécie é de hábito alimentar de fundo, alimentando-se confortavelmente em ambiente escuro. Assim, em situação de ausência de luz, esse tipo de peixe apresenta menor estresse e, consequentemente, melhor desenvolvimento (Appelbaum e McGeer, 1998). Além disso, de acordo com Feiden et al. (2006), alguns siluriformes apresentam fototaxia negativa, habitando, em geral, o fundo dos rios, onde a incidência da luminosidade é baixa. Essas diferenças encontradas na sobrevivência para as diferentes espécies podem ser atribuídas à extrema variação na preferência do fotoperíodo, que é específico de cada espécie, além de depender da fase de desenvolvimento em que se encontra o animal Britz e Pienaar, 1992; SilvaGarcia, 1996; Adewolu et al., 2008; Boeuf e Bail, 1999).

Os resultados apresentados de hematócrito, níveis de glicose sanguínea e cortisol demonstram que não houve situação de estresse crônico devido à manipulação do fotoperíodo, uma vez que essas variáveis se mantiveram constantes nos peixes submetidos a todos os fotoperíodos. Segundo Biswas et al. (2004), tilápias-do-nilo, quando em situação de estresse, apresentam valores de glicose e cortisol de 160,2 $\mathrm{mg} / \mathrm{dL}$ e $191,7 \mathrm{mg} / \mathrm{dL}$, respectivamente. Geralmente o estresse agudo resulta em uma elevação dos níveis de cortisol e glicose em Oreochromis niloticus (Barcellos et al., 1999), assim como em outras espécies de peixes (Barton, 1997; Barton e Iwama, 1991; Pottinger et al., 1999).

Diversos fatores ambientais têm demonstrado serem potenciais fontes de estresse em peixes (Barton, 1997; Biswas et al., 2006a). No entanto, apesar de a manipulação do fotoperíodo trazer certas mudanças, como nos níveis de determinados hormônios e atividade locomotora diária, poucos são os relatos em que o estresse é demonstrado como consequência dessa manipulação (Biswas et al., 2004, 2006a).

Os resultados do hematócrito de alevinos de tilápia foram similares aos demonstrados com a mesma espécie sob os fotoperíodos de 12L:12E e 6L:6E (Biswas et al., 2004). Pesquisas têm demonstrado aumento do hematócrito sob condições de estresse agudo Wang et al., 1994; Pierson et al., 2004), fato este devido ao recrutamento de células vermelhas do baço (Jensen, 1987) e/ou devido ao aumento de tamanho dessas células (Wang et al., 1994). Por outro lado, sob condições de estresse crônico, o hematócrito tem apresentado decréscimo (Barcellos et al., 2004).

No presente estudo, mesmo com a elevada taxa de sobrevivência dos peixes submetidos aos diferentes fotoperíodos, a mortalidade de alguns indivíduos pode ser atribuída ao comportamento agressivo característico da espécie, uma vez que os animais mortos geralmente eram os menores e apresentavam lesões de agressão no corpo e nadadeiras. Estudo com jundiá, Rhamdia quelen, demonstraram que o aumento da luminosidade induz os animais à maior agressividade (Piaia $e t$ al., 1999). Segundo Almazán-Rueda et al. (2005), foi demonstrado maior nível de cortisol e agressividade em bagres africanos (Clarias gariepinus) sob regime de 12L e 18L em relação aos períodos de 6L e 0L. Após dois meses de experimento com truta arco-íris Oncorhynchus mykiss, foi demonstrado que os peixes submetidos ao fotoperíodo artificial de $24 \mathrm{~L}$ apresentaram uma resposta ao estresse com significativa elevação dos níveis plasmáticos de cortisol (Leonardi e Klempau, 2003).

Regimes longos ou constantes de luz também têm demonstrado efeitos negativos no desenvolvimento de larvas de várias espécies (Villamizar et al., 2011). Segundo Villamizar et al. (2009), larvas de sea bass Europeu Dicentrarchus labrax, mantidas em condições de luz constante $(24 \mathrm{~L})$, desenvolvem nadadeiras e dentes mais rápido do que as sob condições de ausência de luz (0L) e fotoperíodo natural (12L). No entanto, embora o regime de luz constante proporcione o melhor crescimento das larvas, as mesmas não apresentavam uma adequada inflação da bexiga natatória, o que é importante, sobretudo à noite, quando as larvas dirigem-se à superfície para apanhar ar e escapar de potenciais 
predadores. Acrescido a esse fato, os mesmos autores ainda relatam a expressiva porcentagem de indivíduos que apresentavam deformações na mandíbula sob essas condições de luz constante. Resultado semelhante foi demonstrado com larvas de Senegal sole Solea senegalensis mantidas em constante condição de luz, as quais apresentaram elevada proporção de má-formação da mandíbula quando comparadas com as mantidas sob o fotoperíodo de 12L (BlancoVives et al., 2010). Além disso, os mesmos autores ainda demonstraram alta taxa de mortalidade das larvas mantidas sob $24 \mathrm{~L}$ e $0 \mathrm{~L}$ quando comparadas com as do fotoperíodo de 12L.

Por outro lado, Biswas et al. (2004), em estudos com tilápia-do-nilo submetidas ao fotoperíodo de 12L:12E e 6L:6E, não verificaram alterações nos parâmetros de glicose, cortisol, hematócrioto e íons cloreto. Estudos com alevinos e adultos de Pagrus major (Biswas et al., 2006a,b) e Oplegnathus fasciatus (Biswas et al., 2008) submetidos aos fotoperíodos de 6L:6E, 12L, 16L e 24L também não demonstraram alterações nos níveis de glicose, cortisol, hematócrito e proteínas totais. Portanto, nessas espécies, assim como em alevinos de tilápia-do-nilo do presente estudo, a manipulação do fotoperíodo não demonstrou interferir nos parâmetros fisiológicos relacionados ao estresse.

\section{CONCLUSÕES}

A manipulação do fotoperíodo, aparentemente, não interfere sobre a homeostase de alevinos de tilápia-do-nilo, uma vez que não influenciou na sobrevivência dos peixes submetidos aos diferentes fotoperíodos, assim como as variáveis fisiológicas relacionadas ao estresse mantiveramse dentro dos padrões pré-determinados para a espécie.

\section{AGRADECIMENTOS}

Ao Conselho Nacional de Pesquisa (CNPq) e à Fundação de Amparo à Pesquisa do Estado de Minas Gerais (FAPEMIG), pelo apoio financeiro para a realização da pesquisa.

\section{REFERÊNCIAS}

AUDET, C.; FITZ GERALD, G.J.; GUDERLEY, H. Photoperiod effects on plasma cortisol levels in Gasterosteus aculeatus. Gen. Comp. Endocrinol, v.61, p.76-81, 1986.

ADEWOLU, M.A.; ADENIJI, C.A.; ADEJOBI, A.B. Feed utilization, growth and survival of Clarias gariepinus (Burchell 1822) fingerlings cultured under different photoperiods. Aquaculture, v.283, p.64-67, 2008.

ALMAZÁN-RUEDA, P.; VAN HELMOND, A.T.M.; VERRETH, J.A.J.; SCHRAMA, J.W. Photoperiod affects growth, behaviour and stress variables in Clarias gariepinus. J. Fish Biol., v.67, p.1029-1039, 2005.

APPELBAUM, S.; MCGEER, J.C. Effect of diet and light regime on growth and survival of African catfish (Clarias gariepinus) larvae and early juveniles. Aquac. Nutr., v.4, p.157-164.

BARCELLOS, L.J.G.; NICOLAIEWSKY, S.; SOUZA, S.M.G.; LULHIER F. Plasmatic levels of cortisol in the response to acute stress in Nile tilapia, Oreochromis niloticus (L.), previously exposed to chronic stress. Aquac. Res., v.30, p.437-444, 1999.

BARCELLOS, L.J.G.; KREUTZ, L.C.; SOUZA, C. et al. Hematological changes in jundiá (Rhamdia quelen Quoy and Gaimard Pimelodidae) after acute and chronic stress caused by usual aquacultural management, with emphasis on immunosuppressive effects. Aquaculture, v.237, p.229-236, 2004.

BARTON, B.A.; IWAMA, G.K. Physiological changes in fish from stress in aquaculture with emphasis on the response and effects of corticosteroids. Annu. Rev. Fish Dis., v.1, p.3-26, 1991.

BARTON, B.A. Stress in finfish: past, present and future - a historical perspective. In: IWAMA, G.K.; PICKERING, A.D.; SUMPTER, J.P.; SCHRECK, C.B. (Eds.). Fish stress and Health in Aquaculture. Society for Experimental Biology Seminar Series 62. Cambridge: Cambridge University Press, v.62, p.1-33, 1997.

BEZERRA, K.S.; SANTOS, A.J.G.; LEITE, M.R. et al. Crescimento e sobrevivência da tilápia chitralada submetida a diferentes fotoperíodos. Pesq. Agropec. Bras., v.43, p.737743, 2008. 
BISWAS, A.K.; ENDO, M.; TAKEUCHI, T. Effect of different photoperiod cycles on metabolic rate and energy loss of both fed and unfed young tilapia Oreochromis niloticus: Part I. Fish. Sci., v.68, p.465-477, 2002.

BISWAS A.K.; TAKEUCHI, T. Effects of different photoperiod cycles on metabolic rate and energy loss of both fed and unfed adult tilapia Oreochromis niloticus: part II. Fish. Sci., v.68, p.543-553, 2002.

BISWAS, A.K.; MAITA, M.; YOSHIZAKI, G.; TAKEUCHI, T. Physiological responses in Nile tilapia exposed to different photoperiod regimes. J. Fish Biol., v.65, p.811-821, 2004.

BISWAS, A.K.; MANABU, S.; KENJI, T. et al. Stress response of red sea bream Pagrus major to acute handling and chronic photoperiod manipulation. Aquaculture, v.252, p.566-572, 2006a.

BISWAS, A.K.; SEOKA, M.; TANAKA, Y. et al. Effect of photoperiod manipulation on the growth performance and stress response of juvenile red sea bream (Pagrus major). Aquaculture, v.258, p.350-356, 2006b.

BISWAS, A.; SEOKA, M.; UENO, K. et al. Growth performance and physiological responses in striped knifejaw, Oplegnathus fasciatus, held under different photoperiods. Aquaculture, v.279, p.42-46, 2008.

BLANCO-VIVES, B.; VILLAMIZAR, N.; RAMOS, J. et al. Effect of daily thermo and photo-cycles of different light spectrum on the development of Senegal sole (Solea senegalensis) larvae. Aquaculture, v.306, p.137$145,2010$.

BOEUF, G.; BAIL, P.Y.L. Does light have an influence on fish growth? Aquaculture, v.177, p.129-152, 1999.

BRITZ, P.J.; PIENAAR, A.G. Laboratory experiments on the effect of light and cover on the behaviour and growth of African catfish, Clarias gariepinus (Pisces: Clariidae). J. Zool., v.227, p.43-62, 1992.

CRUZ, E.M.V.; BROWN, C.L. Influence of the photoperiod on growth rate and insulin-like growth factor-I gene expression in Nile tilapia Oreochromis niloticus. J. Fish Biol., v.75, p.130$141,2009$.
DABROWSKI, K. The point of no return in early fish life. An attempt to determine the minimal food requirement. Wiad. Ekol, Warszawa, v.21, p.277-293, 1975.

EL-SAYED, A.F.M.; KAWANNA, M. Effects of photoperiod on the performance of farmed Nile tilapia Oreochromis niloticus. I-Growth, feed utilization efficiency and survival of fry and fingerlings. Aquaculture, v.231, p.393-402, 2004.

FEIDEN, A.; HAYASHI, C.; BOSCOLO, W.R.; REIDEL, A. Desenvolvimento de larvas de Steindachneridion sp. em diferentes condições de refúgio e luminosidade. Pesq. Agropec. Bras., v.41, p.133-137, 2006.

HOLMES, R.A.; GIBSON, R.W. Visual cues determining prey selection by the turbot, Scophthalmus maximus L. J. Fish Biol., v.29 (suppl. A), p.49-58, 1986.

JENSEN, F.B. Influences of exercise-stress and adrenaline upon intra- and extracellular acid-base status, electrolyte composition and respiratory properties of blood in tench (Tinca tinca) at different seasons. J. Comp. Physiol. B, v.157, p.51-60, 1987.

LARSON, E.T.; WINBERG, S.; MAYER, I. et al. Stress affects circulating melatonin levels in rainbow trout. Gen. Comp. Endoc., v.136, p.322$327,2004$.

LEONARDI, M.O; KLEMPAU, A.E. Artificial photoperiod influence on the immunesystem of juvenile rainbow trout (Oncorhynchus mykiss) in the southern hemisphere. Aquaculture, v.221, p.581-591, 2003.

MENDONÇA, P.P.; FERREIRA, R.A.; VIDAL JUNIOR, M.V. et al. Influência do fotoperíodo no desenvolvimento de juvenis de tambaqui (Colosssoma macropomum). Arch. Zootec., v.58, p.323-331, 2009.

PIAIA, R.; TOWSEND, C.R; BALDISSEROTTO, B. Growth and survival of fingerlings of Rhamdia quelen exposed to different light regimes. Aquac. Internat., v.7, p.201-205, 1999.

PICKERING, A.D.; POTTINGER, T.G. Seasonal and diel changes in plasma cortisol levels of brown trout, Salmo trutta L. Gen. Comp. Endocrinol. v.49, p.232-239, 1983. 
PIERSON, P.M.; LAMERS, A.; FLIK， G.; MAYER-GOSTAN, N. The stress axis, stanniocalcin, and ion balance in rainbow trout. Gen. Comp. Endoc., v.137, p.665-678, 2004.

RAD, F.; BOZAOĞLU, S.; GÖZÜKARA, S.E.; KARAHAN, A. et al. Effects of different longday photoperiods on somatic growth and gonadal development in Nile tilapia (Oreochromis niloticus L.). Aquaculture, v.255, p.292-300, 2006.

SHAN, X.; XIAO, Z.; HUANG, W.; DOU, S. Effects of photoperiod on growth, mortality and digestive enzymes in miiuy croaker larvae and juveniles. Aquaculture, v.281, p.70-76, 2008.

SILVA-GARCIA, A.J. Growth of juvenile gilthead seabream (Sparus aurata L.) reared under different photoperiod regimes. Isr. J. Aquacuacult-Bamid., v.48, p.84-93, 1996.

TRIPPEL, E.A.; NEIL, S.R.E. Effects of photoperiod and light intensity on growth and activity of juvenile haddock (Melanogrammus aeglefinus). Aquaculture, v.217, p.633-645, 2003.
VILLAMIZAR, N.; GARCÍA-ALCAZAR, A.; SÁNCHEZ-VÁZQUEZ, F.J. Effect of light spectrum and photoperiod on the growth, development and survival of European sea bass (Dicentrarchus labrax) larvae. Aquaculture, v.292, p.80-86, 2009.

VILLAMIZAR, N.; BLANCO-VIVES, B.; MIGAUD, H. et al. Effects of light during early larval development of some aquacultured teleosts: A review. Aquaculture, v.315, p.86-94, 2011.

WANG, Y.; HEIGENHAUSER, G.J.; WOOD, C.M. Integrated responses to exhaustive exercise and recovery in rainbow trout white muscle: acid-base, phosphogen, carbohydrate, lipid, ammonia, fluid volume and electrolyte metabolism. J. Exp. Biol., v.195, p.227-258, 1994.

WENDELAAR BONGA, S.E. The stress response in fish. Physiol. Rev., v.77, p.591-625, 1997. 